\title{
Current Perspectives in Meniere's Disease Treatment: A Mini Review
}

\author{
Georgios K. Panagiotopoulos* MD MSc MAud/Notol ASCP(VSD) AuD PhD
}

${ }^{1}$ Athens Balance \& Hearing center, Private ENT practice, Greece.

*Corresponding author: Georgios K Panagiotopoulos, OtoRhinoLaryngologist Neurotologist, Leoforos Pentelis 37A Vrilissia, PC 15235, Athens, Greece.

\begin{abstract}
Meniere's disease (MD) is a chronic heterogeneous disorder recently found to involve multiple rare and common variants in several genes. It almost always necessitates management for a sustained period of time. Treatment options focus on improving quality of life in MD patients. Control is not always easy to achieve, requiring multidrug treatments, and even surgical procedures. Migraine, dyslipidemia, obesity, diabetes, sleep apnea, hypertension, and atherosclerosis are among the major diseases that are associated with MD and often require medical management. Migraine prophylaxis and trigger elimination, magnesium supplementation, low sodium intake, diuretics, anticoagulants, and antihypertensives are among the treatments used initially. Steroids administered orally or intratympanically are used if medical control is not beneficial. Ablation remains the definitive treatment in unilateral cases experiencing management failure, thus facilitating a customized vestibular rehabilitation protocol. Genetic study should be implemented especially when familial MD is considered. Proper gene variance targeting in MD can provide in the immediate future candidates for potential gene therapy.
\end{abstract}

Keywords: Meniere's disease; Treatment; Gene therapy

\section{Introduction}

Meniere's disease (MD) is considered as an heterogeneous group of disorders defined by 3 major symptoms: early onset fluctuating sensorineural hearing loss affecting low to mid frequencies, episodic vertigo and tinnitus/aural fullness [1]. Although published temporal bone studies suggest that MD has characteristic signs of endolymphatic hydrops, saccular endolymphatic hydrops can also be found in $10 \%$ of normal subjects and in $40 \%$ patients with $>45 \mathrm{~dB}$ sensorineural hearing loss without any sign of vestibular involvement [2].

MD symptoms are thought to be caused by the accumulation of endolymph in the membranous labyrinth, with consequent endolymphatic hypertension, a finding that has been demonstrated in anatomical and histopathological studies [3]. Endolymphatic hypertension leads to dysfunction and consequently damage of the hair cells located in the anterior and posterior labyrinth. MD is a multifactorial disorder and involves the participation of genetic (autosomal dominant or recessive pattern of inheritance with incomplete penetrance and variable expressivity) and external factors [1]. MD also shows comorbidities with several other disorders including autoimmune diseases (especially in bilateral MD cases), allergy and migraine [1], a fact that should be taken into account during our interventions.

\section{Treatment Basics}

Treatment options focus on improving quality of life of MD patients. An individualized approach for MD patients is strongly recommended. If a comorbid condition such as allergy, migraine or autoimmune disorder is present, then it should be treated accordingly. Besides that, treatment of MD symptoms is based initially on the control and hopefully the reversal of endolymphatic hypertension which can be done through change in life habits, diet accommodations, drugs and others [3]. Such non-surgical interventions try to normalize the membranous inner ear 
homeostasis and hopefully control the natural evolution of the disease as well as its symptomatology. When symptom relief cannot be achieved or sustained by conservative treatments, one may consider using more invasive procedures that may assist either in controlling endolymphatic hypertension, or destroying the sensory epithelium itself, thus alleviating the symptoms.

\section{Dietary Modifications}

Numerous studies [3] suggest that water retention, due to a potential increase of body's sodium levels, may induce hydrops and thus exacerbate the symptoms of MD. This is the rationale behind the restriction of salt, as a way to control symptomatology in patients with MD. A low sodium diet can increase plasma concentration of aldosterone, which can enable the transport of ions in favor of the absorption of endolymph inside the endolymphatic sac. It is worth noting that the effectiveness of this intervention is shown when sodium intake is reduced to less than $3 \mathrm{~g}$ per day [3]. Nutrition education by referral to a dietitian can achieve such a goal thus improving outcomes in the pre-medical treatment of MD. Other dietary changes focus on limiting alcohol and caffeine intake [4]. This relies on the fact that both alcohol and caffeine can lead to vasoconstriction and therefore decrease the blood supply to the labyrinth, which can further worsen the symptomatology of patients.

\section{Oral Drugs}

\section{Histamine and related medications-betahistine}

H1 receptor blockers and calcium antagonist, (such as flunarizine and cinnarizine), inhibit vasoconstriction and act as vestibular sedatives [3]. They can be beneficial in the treatment of vertigo of central and peripheral origin. Both are contraindicated in patients with extrapyramidal disorders [3], being useful for symptomatic only management and mainly during vertigo attacks.

Betahistine is a weak histamine $\mathrm{H} 1$ agonist and a stronger $\mathrm{H} 3$ antagonist. It is generally well tolerated with a low risk of adverse events. Betahistine is widely used to reduce the frequency and severity of the vertiginous attacks of MD but there is conflicting evidence relating to its effects [3]. A positive effect of betahistine in terms of reduction in vertigo symptoms is attributed to endolymph pressure reduction secondary to microcirculation dilatation at the stria vascularis of the cochlea. Another proposed mechanism relates to vestibular nucleus activity inhibition [5]. Recent metanalyses point out that, although betahistine might reduce the number of vertigo attacks and their intensity, potentially leading to subjective improvement of symptoms, the evidence is rather poor $[5,6]$.

\section{Gingko biloba (EGb 761)}

This natural extract is widely used in the treatment of vertigo of peripheral origin [3]. Similarly to betahistine, although it is considered as being better tolerated by the majority of the patients, there isn't adequate high level evidence to support its efficacy in the treatment of vertigo in MD. Headache, low blood pressure, and gastrointestinal disturbances are the main side effects that are associated with high doses of Gingko Biloba administration.

\section{Benzodiazepines}

The benzodiazepines are a family of sedating medications used for treatment of both anxiety and vertigo. Commonly prescribed benzodiazepines include among others diazepam, lorazepam, clonazepam and alprazolam. These drugs are largely used to treat vertigo related dysphoria, but also are used to induce sleep, mainly by suppression of the central nervous system. They are best used in small doses, and ideally for a limited time (routinely less than one month). Generally, benzodiazepines are not toxic, in the sense that they do not tend to damage organ systems, but on the other hand they are associated with addiction and tolerance. They are beneficial in alleviating the severity of symptoms of sporadic vertigo attacks in MD. They act by decreasing the activity of the vestibular apparatus more centrally (they increase the inhibitory effect of gamma-aminobutyric acid at the level of the vestibular nuclei). They are therefore useful in the therapy of acute vertigo, along with the control of distress, and panic attacks in patients with MD. Common side effects include drowsiness, fatigue, and more importantly drug dependence.

\section{Diuretics}

Diuretics can alter the balance of electrolytes in the inner ear, thus reducing the volume and pressure of the endolymph. This can take place either by improved drainage of the endolymph itself or by reduced production in the first place [3]. They are usually used to control vertigo attacks, audiometric threshold shifts, tinnitus, and aural fullness in MD patients. Recent systematic review [7] concludes that there is a low-level evidence regarding the improvement in vertigo episode frequency, with even less convincing evidence for improvement in hearing outcomes themselves. This review takes into consideration the various diuretics most widely used for the medical management of MD; a combination of hydrochlorothiazide and triamterene is the most commonly used medical treatment for MD; acetazolamide, furosemide, and spironolactone are less preferred by others [8]. Diuretics' common side effects are associated with alterations in the concentration of the electrolytes in the blood (mainly potassium and sodium).

\section{Intratympanic Medications}

The aim of these treatments is to utilize the medication that may potentially affect the function of the cochlea and the labyrinth, directly by entering the inner ear through the round window [3]. Intratympanic treatment is considered to be a very popular intervention as it is very practical and easy to apply, even in the office setup, with minor complications. On the other hand, systemic delivery of various medications destined for the treatment of an inner ear dysfunction is far less popular due to presence of systemic side effects, that can be easily avoided by utilizing techniques to deliver therapeutic agents locally. For instance, systemic steroid administration is well associated with many side effects including 
hyperglycemia, hypokalemia, gastric disease, hypertension, osteoporosis, immunosuppression, and, if given over a sustained period of time, adrenal suppression [9]. That is why delivery of medications directly to the inner ear has been an area of remarkable growth in both the research and clinical realms over the last several decades.

\section{Methods for intratympanic delivery}

There are different protocols published regarding the optimum intratympanic administration series although the difference between the various protocols still remains to be properly evaluated. The simplest and quickest form is via injection into the middle ear either directly with a needle or through a myringotomy with or without a ventilation tube [9]. Transtympanic injection routinely require the need for repeat procedures should dosing of medication exceeds one-time application basis. On the other hand, any method that involves a placement of a tympanostomy tube or a similarly made communication between the outer and middle ear can provide the inner ear with sustained release of medication over a longer period of time. Such interventions come with a small, but real, risk of persistent perforation of the tympanic membrane upon extrusion of the tube, infection of the mucosa of the middle ear associated with the either presence of the tube or the open contact between the outer and middle part of the ear, and the need to constantly keep the ear dry to avoid further complications.

The Silverstein Micro Wick and implantable microcatheters [9] are alternative systems with capability to deliver medication to the middle ear continuously over a period of several weeks as needed. Recent advantages in technology also encompass the utilization of hydrogel and nanoparticles [9] (in the size range of 200nm or less) in order to deliver consistent doses of medication to the inner ear via the round window in a controlled fashion and over longer period of time.

\section{Corticosteroids}

Corticosteroids are able to reduce the inflammation in the ear and potentially increase inner ear circulation. Moreover, evidence suggests that the steroids can beneficially affect the metabolism of sodium inside the endolymph [3]. Between the two available steroids derivatives available, dexamethasone versus methylprednisolone, the former is considered as more practical to use. Dexamethasone exhibits far better tolerance by the patients, as methylprednisolone is strongly associated with a burning sensation in the middle ear mucosa. The drawback with dexamethasone is its present availability with low concentrations, such as $4 \mathrm{mg} / \mathrm{mL}^{3}$ as it is believed that higher concentrations can produce stronger and more lasting effects.

\section{Gentamicin}

The rationale behind this therapy is to cause a permanent chemical damage to the sensory epithelium, in order to stop the fluctuating labyrinth malfunction. These fluctuations are responsible for the vertigo attacks in MD that can't be easily controlled by the brain's own plasticity mechanisms. Simply by creating a stable situation of paresis or hypoactivity the brain can re-adjust and compensate with the help of a targeted vestibular rehabilitation program. This treatment can only decrease the episodes of dizziness in Meniere's disease and it may leave a permanent hearing loss.

\section{Interventions to Relieve Distention of the Endolymphatic System}

Endolymphatic shunts of various kinds, cochleosacculotomy, and endolymphatic sac decompressions have been used for many decades [8]. There have been several discussions in favor and against endolymphatic sac surgery. The evidence level to support this surgery is low and the potential benefit controversial [1]. During the procedure a stent is placed inside the sac that directs the excess of endolymph towards the mastoid cavity or the cerebrospinal fluid compartment. Based on the same surgical principle, similar results are achieved with decompression of the endolymphatic sac against shunting maneuvers [3]. Recent approaches [1] advocated the direct injection of dexamethasone into the sac. There are suggestions that endolymphatic sac shunt procedures may benefit from steroid instillation at the time of shunt placement, based on the fact that the endolymphatic sac is the only location for immune reactions in the temporal bone [10].

Shunt procedures are still popular and represent the major conservative surgical treatment for the disorder. Over $80 \%$ of otologists tend to use shunts for medically refractory MD $[8,10]$. They are also a viable option for patients with MD affecting both ears, as ablative procedures cannot be used bilaterally in such group of patients. Nevertheless, there is no evidence that shunts can influence in any way the natural history of the disease [8].

\section{Meniett Device}

Early 1970's publications reported that some patients experienced improvements regarding their balance and hearing status with changes of pressure in a pressure chamber. In more detail, it was observed that the manipulation of the middle ear pressure could influence the pressure in the inner ear and consequently improve hearing and dizziness in patients with MD. These alterations were noted after the application of positive pressure in the middle ear along with an improvement of the cochlear electrical potentials. These findings finally led to the development of the device known as the Meniett [3], which emits a pulse of repeated pressure of 0.6 second in the range of up to $20 \mathrm{~cm}$ $\mathrm{H} 2 \mathrm{O}$ at $6 \mathrm{~Hz}$. Treatment consists of three to four cycles of a sequence of treatment of $5 \mathrm{~min}$, and it requires only a short-term ventilation tube in order to allow the transmission of impulses through the external part of the ear to its middle part. Although the FDA approved the use of the device in 2002, and despite recent work showing the effectiveness of the device [3] (in terms of reducing the frequency of dizziness in MD patients), it does not significantly 
improve hearing, becoming just a safe alternative for some patients.

\section{Advanced Surgery}

\section{Labyrinthectomy}

Labyrinthectomy is the oldest surgical method to treat MD, and today is limited to older patients. The goal of this surgical approach is the complete removal of the five vestibular end organs: the three semicircular canals and the two otolith organs; the utricule and the saccule. In patients with severe to profound hearing loss who do not respond to other surgical and medical treatments, labyrinthectomy is still a liable alternative surgical option for unilateral MD. Bilateral MD on the other hand is a contraindication for this procedure, because of the related oscillopsia and permanent imbalance that can result from bilateral vestibular loss [3]. For patients with unilateral MD and profound sensorineural hearing loss, a simultaneous labyrinthectomy along with cochlear implantation [3] is thought to be an efficient alternative, focusing on the treatment of vertigo and the optimum rehabilitation of the hearing loss.

\section{Vestibular nerve section (VNS)-vestibular neurectomy}

Vestibular nerve sectioning is one of the most effective procedures for treating persistent vertigo in patients suffering from unilateral MD. In the literature [3], vertigo control is reported in 80$90 \%$ of cases. Vestibular neurectomy can be selectively targeted to either the superior or inferior branch of the vestibular nerve trying to maintain the cochlear nerve intact.

\section{Gene Therapy}

Meniere disease (MD) represents a complex disease recently found to involve multiple rare and common variants in several genes [11-13]. Current evidence [12,13] supports a genetic contribution in MD in an attempt to decipher the genetic architecture and to design and develop a molecular map of MD. Genetic study should be implemented especially when familial MD is considered (as it is observed in 8-9\% of sporadic cases in Europe) [11]. Proper gene variance targeting can provide in the immediate future candidates for potential gene therapy.

\section{Discussion}

MD is a chronic heterogeneous condition that requires treatment for long time and whose control is not always easy to achieve, requiring some multidrug treatments, and sometimes even surgical procedures.

Migraine, dyslipidemia, obesity, diabetes, sleep apnea, hypertension, and atherosclerosis are among the major diseases that are associated with MD and often require medical management. Migraine prophylaxis and trigger elimination, magnesium supplementation, low sodium intake, diuretics, anticoagulants, and antihypertensives are among the treatments used initially. Steroids administered orally or intratympanically are used if medical control is below average, and ablation remains the definitive treatment in unilateral cases experiencing management failure [8].
With regards to neurectomy, the intratympanic administration of gentamicin has received far more interest due to its strong effect over the vertigo episodes, beating down the frequency of vestibular neurectomies. Nevertheless, neurectomy is still believed to be the most efficient technique for addressing drop attacks (Tumarkin's disorder) and for incapacitating Ménière disease. It must be emphasized that vestibular rehabilitation therapy is quite effective only for patients with stable vestibular (hypo-) function in order to improve not only vestibular function but also central mechanisms of adaptation, substitution and compensation.

\section{Conclusion}

Although there are many pharmaceutical options and surgical interventions available for the treatment of MD, the final treatment plan should be strictly customized. Such plan should take into consideration not only the stage of the disease but also individual features related to the patient's own beliefs and way of living. Genetic variance targeting is a promising addition to our current arsenal of management options, as it can provide in the immediate future candidates for potential gene therapy.

\section{Acknowledgements}

None.

\section{Conflict of Interest}

None.

\section{References}

1. Magnan J, Özgirgin ON, Trabalzini F, Lacour M, Escamez AL, et al. (2018) European Position Statement on Diagnosis, and Treatment of Meniere's Disease. J Int Adv Otol 14(2): 317-321.

2. Attyé, A, Eliezer M, Medici M, Tropres I, Dumas G, et al. (2018) In vivo imaging of saccular hydrops in humans reflects sensorineural hearing loss rather than Meniere's disease symptoms. Eur Radio: 1-7.

3. Bogaz EA, da Silva, AFC, Ribeiro DK, dos Santos Freitas G (2017) Meniere's Disease Treatment. In Up to Date on Meniere's Disease. InTech.

4. Luxford E, Berliner KI, Lee J, Luxford WM (2013) Dietary modification as adjunct treatment in Ménière's disease: Patient willingness and ability to comply. Otol Neurotol 34(8): 1438-1443.

5. Rosenbaum A, Winter M (2017) Is betahistine effective for Ménière's disease? Medwave 17(08)

6. Adrion C, Fischer CS, Wagner J, Gürkov R, Mansmann U, et al. (2016) Efficacy and safety of betahistine treatment in patients with Meniere's disease: primary results of a long term, multicentre, double blind, randomised, placebo controlled, dose defining trial (BEMED trial). bmj 352 h6816.

7. Crowson MG, Patki A, Tucci DL (2016) A systematic review of diuretics in the medical management of Ménière's disease. Otolaryngology--Head and Neck Surgery 154(5): 824-834

8. Foster CA (2015) Optimal management of Ménière's disease. Ther Clin Risk Manag 11: 301-307.

9. McCall AA, Swan EEL, Borenstein JT, Sewell WF, Kujawa SG, et al. (2010) Drug delivery for treatment of inner ear disease: current state of knowledge. Ear Hear 31(2): 156-165.

10. Wick CC, Manzoor NF, McKenna C, Semaan MT, Megerian CA (2017) Long-term outcomes of endolymphatic sac shunting with local steroids for Meniere's disease. Am J Otolaryngol 38(3): 285-290. 
11. Requena T, Espinosa-Sanchez JM, Cabrera S, Trinidad G, Soto Varela A et al. (2014) Familial clustering and genetic heterogeneity in Meniere's disease. Clin genet 85(3): 245-252.

12. Gallego-Martinez A, Lopez-Escamez J A (2019) Genetic architecture of Meniere's disease. Hear Res 107872.
13. Roman-Naranjo P, Gallego-Martinez A, Soto-Varela A, Aran I, del Carmen Moleon M, et al. (2020) Burden of rare variants in the OTOG gene in familial Meniere's disease. Ear Hear 41(6): 1598-1605. 